\title{
Prognostic Significance of Elevated Cardiac Troponin in Acute Gastrointestinal Bleeding
}

\author{
Umair Iqbala, d, Osama Siddique $^{\mathrm{b}}$, Ayesha Jameel ${ }^{\mathrm{a}}$, Hafsa Anwarc, \\ Ahmad Chaudhary ${ }^{\text {a }}$
}

\begin{abstract}
Background: Acute gastrointestinal bleeding (AGIB) is responsible for over 140,000 hospitalizations annually. Cardiovascular-related deaths account for $30 \%$ of the patients surviving the initial episode of AGIB. The purpose of this study was to identify the impact of elevated troponin on short-term mortality and length of stay (LOS) of these patients.
\end{abstract}

Methods: From July 2013 to July 2016, 290 patients admitted with a diagnosis of AGIB and who had cardiac troponin I measured within $24 \mathrm{~h}$ of presentation were retrospectively reviewed. Clinical variables including 30-day mortality, 30-day readmission and LOS were then compared between the groups of troponin elevation and no troponin elevation.

Results: The overall 30-day mortality among patients with AGIB was $6.5 \%(19 / 290)$. Cardiac troponin was elevated in $10 \%$ of patients (29/290). Among patients with normal troponin, 5\% (13/261) died within 30 days. In patients with troponin elevation, $21 \%$ died in the same period $(6 / 29, \mathrm{P}=0.001)$. The LOS was also higher in patients with troponin elevation ( 6 vs. 5 days, $\mathrm{P}=0.02$ ). There was no difference in 30-day readmission among the two groups. Past history of coronary artery disease, congestive heart failure, hypertension, aspirin use and elevated creatinine was more common in patients with troponin elevation. On multivariate analysis, troponin elevation on presentation is associated with increased mortality (odds: 5.50, CI: $1.73-17.47, \mathrm{P}=0.004)$.

Conclusion: In patients admitted to the inpatient service with AGIB, elevated troponin I on presentation is associated with high short-term mortality and longer hospital stay.

Keywords: Acute gastrointestinal bleeding; Troponin I; Troponin in gastrointestinal bleeding; Mortality; Length of stay

Manuscript submitted July 28, 2017, accepted August 10, 2017

aBassett Medical Center, One Atwell Road, Cooperstown, NY 13326, USA bMemorial Hospital of Rhode Island/Brown University, Providence, RI, USA ${ }^{c}$ Dow University of Health and Sciences, Karachi, Pakistan

${ }^{\mathrm{d} C}$ Corresponding Author: Umair Iqbal, Bassett Medical Center, One Atwell Road, Cooperstown, NY 13326, USA. Email: umair.iqbal@bassett.org

doi: https://doi.org/10.14740/gr893w

\section{Introduction}

Acute gastrointestinal bleeding (AGIB) is an extremely common inpatient and outpatient clinical problem. There are over 140,000 admissions due to AGIB alone in the US per annum associated with significant morbidity and mortality [1]. In order to stratify patients based on outcomes including deaths, there have been numerous scores that are currently recommended per international guidelines. The more common scores used to stratify patients based on the need for intervention or mortality are Glasgow-Blatchford score (GBS) and Rockall score, respectively. These scores majorly incorporate variables including age, gender, vital signs and comorbidities, with no clinical laboratory variables.

There has been an increasing trend of interventional endoscopic therapy used for brisk AGIB, therefore not surprisingly the mortality from AGIB has halved over the past two decades [2]. Yet the economic burden due to inpatient-stay from AGIB is in excess of one billion dollars per year [1]. With increased focus on efficient management and shorter hospital stay, there is a need to proficiently stratify patients who need intervention within $24 \mathrm{~h}$, so as to reduce morbidity, mortality and length of hospital stay (LOS).

In patients admitted for AGIB, the majority of the deaths unrelated to hemorrhage were from a complication of cardiovascular disease and reported to be as high as $30 \%$ in patients who survived the initial episode of upper GI bleed [3, 4]. AGIB can cause hypotension and hypovolemia, and therefore lead to a diminished blood supply to the heart, causing myocardial injury (MI). The American College of Cardiology (ACC) guidelines has identified troponin as the biomarker of choice for detection of MI [5]. Patients with pre-existing obstructing coronary artery disease (CAD) are at a higher risk of cardiovascular complications.

To predict increased mortality, different predictors have been studied and identified, including hypotension on admission, impaired kidney function, and albumin [6,7]. Cardiac troponin has also been studied as a mortality marker particularly in AGIB patients with a cardiac compromise in ICU. Cardiac troponin I (TnI) is located on the actin filament of the cardiac muscle [8]. Its release in the blood stream is not just specific for MI, but can also be an indicator of hypovolemic shock, acute heart failure, transient loss of membrane integrity, and reperfusion injury, all of which can be a complication of 


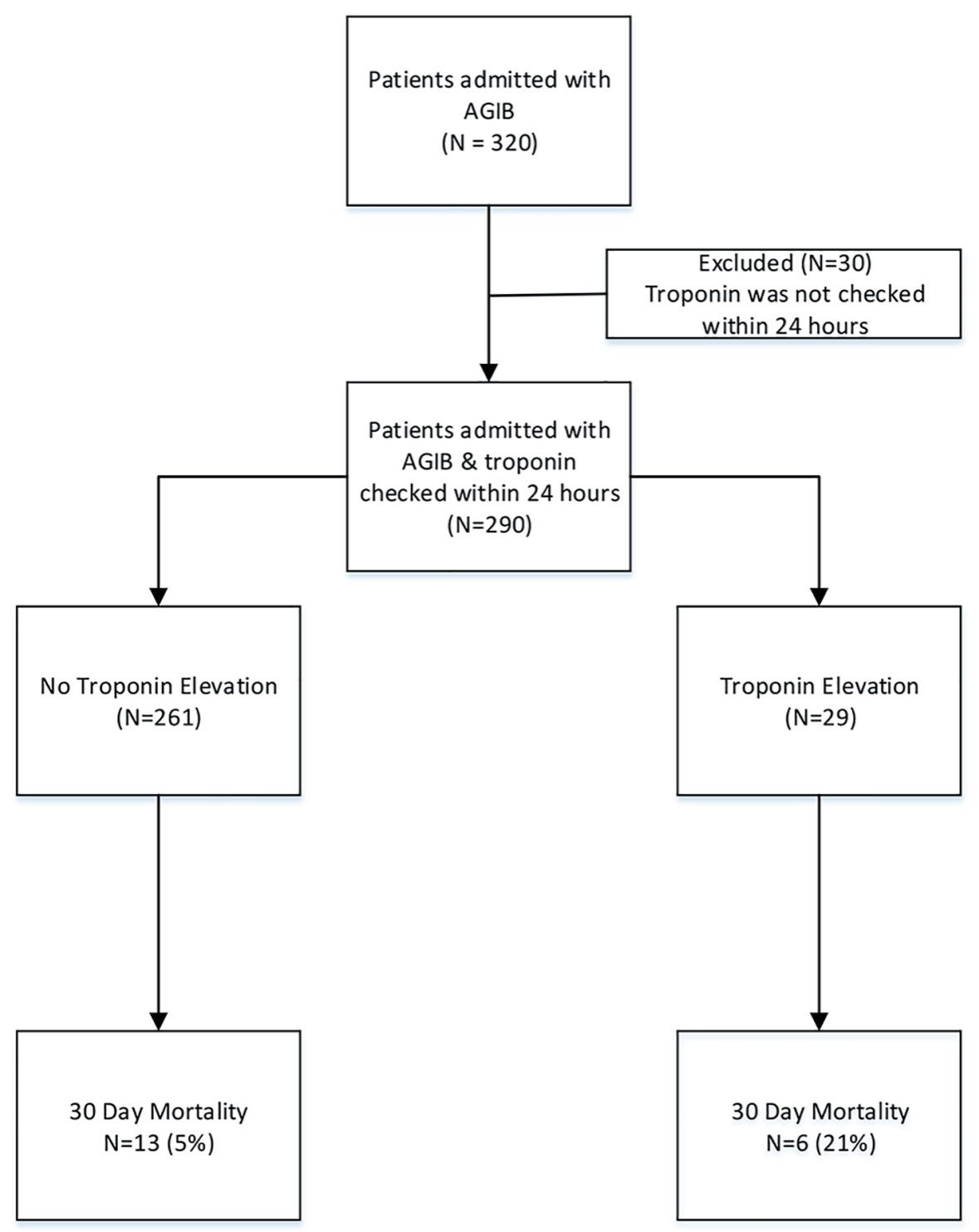

Figure 1. Patients admitted with acute gastrointestinal bleeding (AGIB).

AGIB $[8,9]$. The rise in troponin levels on admission in the ICU with AGIB has been shown to signify a longer hospital stay and higher long-term mortality in patients $[10,11]$, though it has not been widely used to observe any difference in shortterm mortality. Accordingly, the purpose of this study was to evaluate TnI as a prognostic marker for 30-day mortality and its significant impact on LOS in patients admitted with AGIB on the regular floors and ICU. We hypothesized that patients with higher TnI would have a poorer prognosis, independent of their underlying comorbidities.

\section{Patients and Methods}

We retrospectively reviewed electronic medical records of 320 patients consecutively admitted at Bassett Medical Center, Cooperstown, NY, between July 2013 and July 2016 with diagnosis of AGIB. Two hundred ninety (290) patients had a troponin I levels checked within $24 \mathrm{~h}$ of presentation were included in the study (Fig. 1). The study was approved by the institutional review board of Bassett Medical Center. AGIB was defined as either non-variceal upper GI bleeding, variceal upper GI bleeding or lower GI bleeding. Since this was a retrospective study, patients were recognized by their ICD-9 or ICD-10 codes (530.82, 578.9, K92.2, K90.0, K22.8, and K57.91).

All patients with a complete medical history, physical examination, discharge summary and troponin I measurement within $24 \mathrm{~h}$ of admission were included in the study. Medical histories including cardiovascular compromise, cardiovascular comorbidities, such as previous congestive heart failure (CHF), hypertension and atrial fibrillation with use of anticoagulation were recorded in the study. Risk factors for cardiovascular disease (smoking, diabetes, alcohol and chronic kid- 
Table 1. Univariate Association Between Patients With Troponin Elevation and Normal Troponin

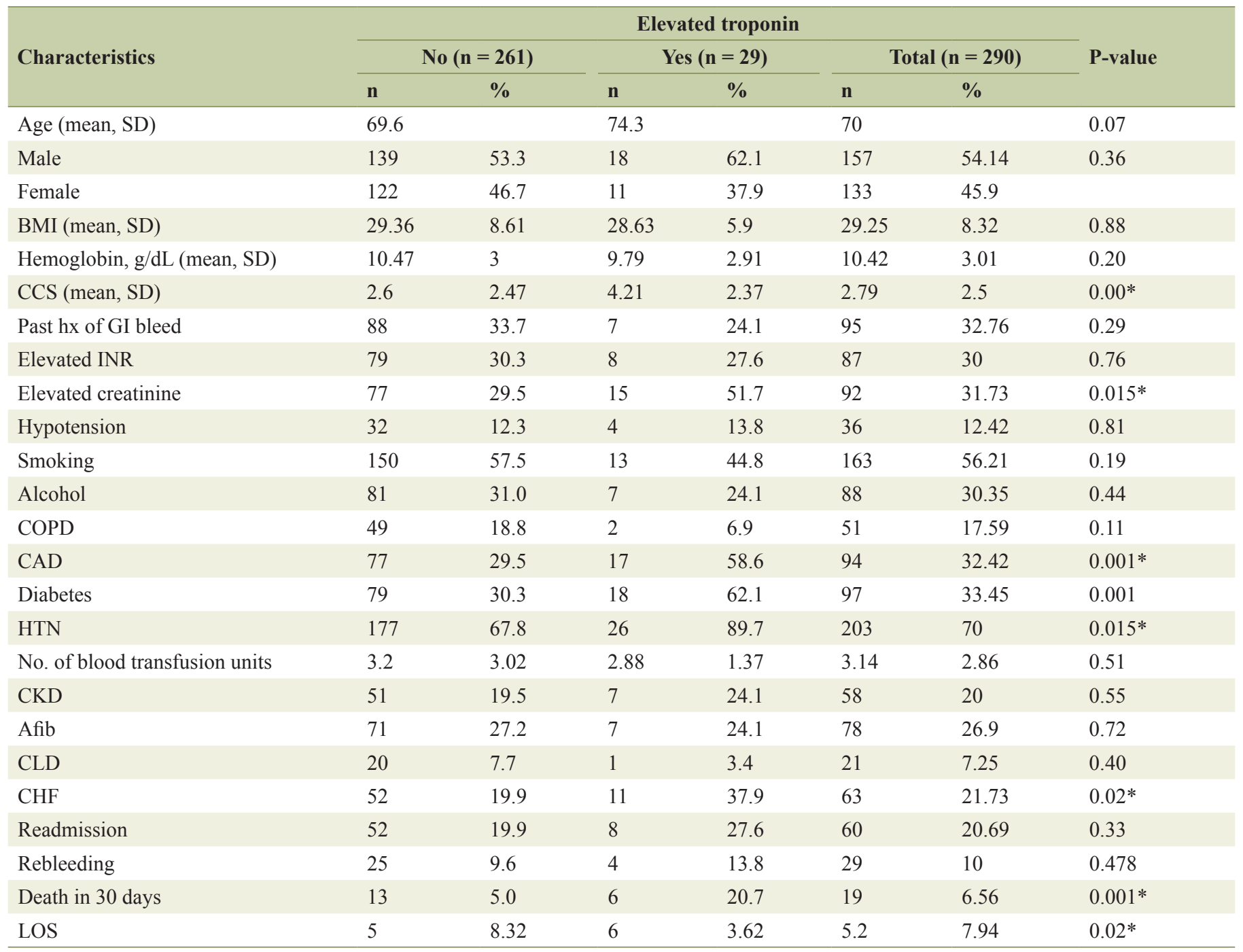

ney disease) were recorded. Patients less than 18 years of age were excluded from the study.

Troponin I levels of $>0.05 \mathrm{ng} / \mathrm{mL}$ were considered elevated. Elevated troponin I levels within $24 \mathrm{~h}$ of admission were used to define patients with cardiovascular damage in accordance with the definition provided by ACC guidelines [5]. Patients were divided into two groups based on troponin I results: patients with elevated troponin, and patients with normal troponin levels. Two hundred sixty-one (261) patients were admitted with normal troponin levels, and 29 had troponin elevation. Statistical analysis was done between the two groups to compare the outcomes including the 30-day mortality, LOS, and the 30-day readmission rate.

\section{Statistical analysis}

To identify univariate associations between troponin elevation and subject characteristics, the Chi-square/Fisher's exact test and $t$-tests were used. $\mathrm{P}$ value of $<0.05$ was considered statistically significant. All variables with $\mathrm{P}<0.2$ in the initial analysis were included in a stepwise multivariable logistic regression model to identify independent predictors of troponin elevation. All analyses were carried out using SPSS 23.0 version.

\section{Results}

There were a total of 320 patients admitted to the hospital from July 2013 to July 2016. Troponin I measurements within $24 \mathrm{~h}$ of admission were available in 290 patients (90\%). The baseline and clinical characteristics in patients who had their troponin I recorded within $24 \mathrm{~h}$ are shown in Table 1 . The median age between the two groups was comparable. Troponin elevation was more in men $(62 \%$ vs. $53 \%, \mathrm{P}=0.36)$ but was non-significant.

The prevalence of troponin I elevation was found in 29 


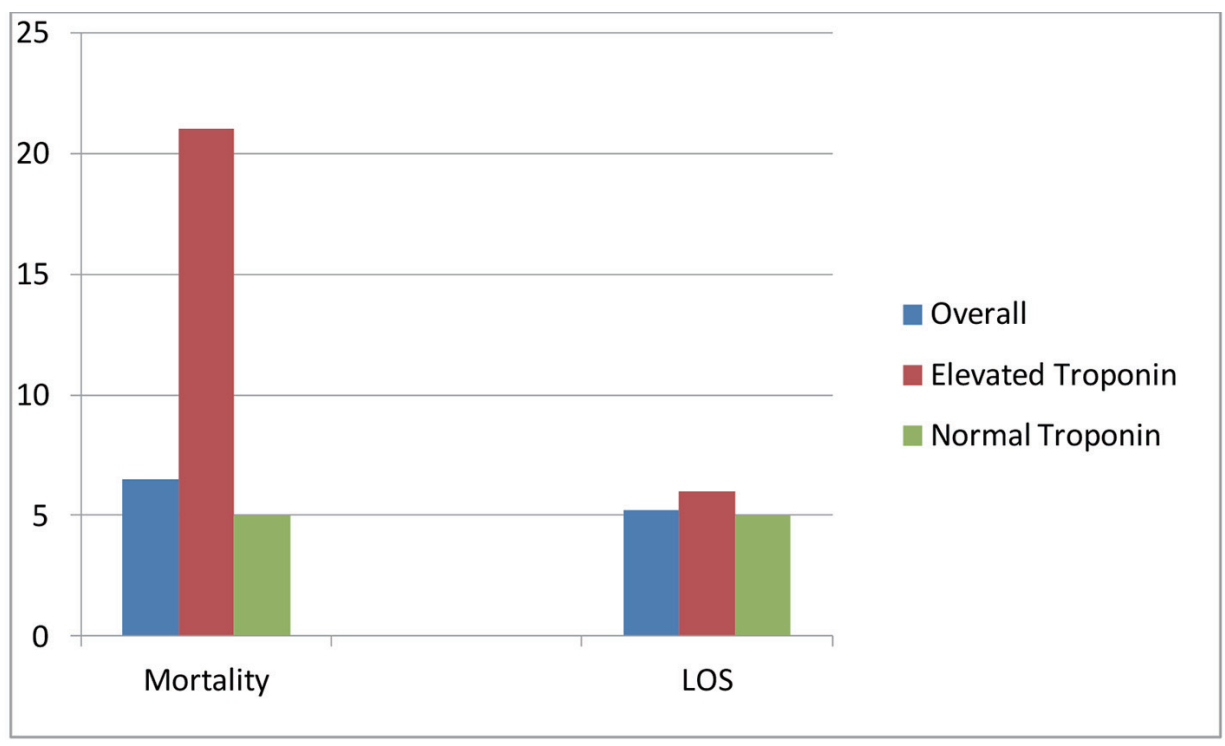

Figure 2. Mortality in \% and length of stay (LOS) in days.

of the 290 patients $(10 \%)$. During the hospitalization, there were 19 deaths amongst the 290 patients (6.5\%). Mortality trended higher in patients with troponin elevation on presentation compared to patients with no troponin elevation; six patients with troponin $\mathrm{I}>0.05 \mathrm{ng} / \mathrm{mL}(20.7 \%)$ versus 13 patients with troponin $\mathrm{I} \leq 0.05 \mathrm{ng} / \mathrm{mL}(5 \%)(\mathrm{P}=0.001)$. The LOS was higher in patients with troponin elevation ( 6 days in troponin elevation group vs. 5 days in no troponin elevation, $\mathrm{P}=0.02$ ) (Fig. 2). The rate of readmission trended higher in the troponin elevation group, but was not statistically significant (27.6\% vs. $19.9 \%, P=0.33)$. There was also no difference in rate of rebleeding between the groups.

Hypotension was defined as systolic BP $<90 \mathrm{~mm} \mathrm{Hg}$ and no statistical difference was found in the hypotension between groups $(13.8 \%$ in troponin elevation vs. $12.3 \%$ in no troponin elevation). Hemoglobin level was lower $(9.8 \mathrm{~g} / \mathrm{dL})$ in the patients with elevated troponin compared to patients with no elevation in their troponin $(10.5 \mathrm{~g} / \mathrm{dL})$; however, it did not reach statistical significance $(\mathrm{P}=0.2)$. Coronary artery disease was more prevalent in the troponin elevation group $(58.6 \%$ vs. $29.5 \%, \mathrm{P}=0.001$ ). Diabetes, hypertension, and CHF were also significantly more prevalent in patient troponin elevation.
The Charlson comorbidity score also trended higher in patients with troponin elevation ( 4.21 vs. $2.6, \mathrm{P}=0.001)$, hence patients with troponin elevation had more comorbidities compared to patients with no troponin elevation. More patients with troponin elevation had elevated creatinine from the baseline (51.7 vs. $29.5 \%, P=0.015)$. Stepwise logistic regression concluded that elevated creatinine is associated with increased odds of troponin elevation.

All parameters with $P$ value of less than 0.2 were thus included in stepwise logistic regression model to predict troponin elevation. Patients with troponin elevation have 5.5 times risk of mortality in 30 days (CI: $1.73-17.47, \mathrm{P}=0.004$ ) (Table 2).

\section{Discussion}

AGIB is a substantial contributor to morbidity, mortality and increased LOS [12]. The main results of the study were that the patients with AGIB and troponin elevation within $24 \mathrm{~h}$ of admission had a higher short-term mortality (defined as 30day mortality) and longer LOS when compared to patients who had no troponin elevation. In this respect, comparative

Table 2. Multivariate Logistic Regression Revealed High Odds of Mortality in Patient With Troponin Elevation

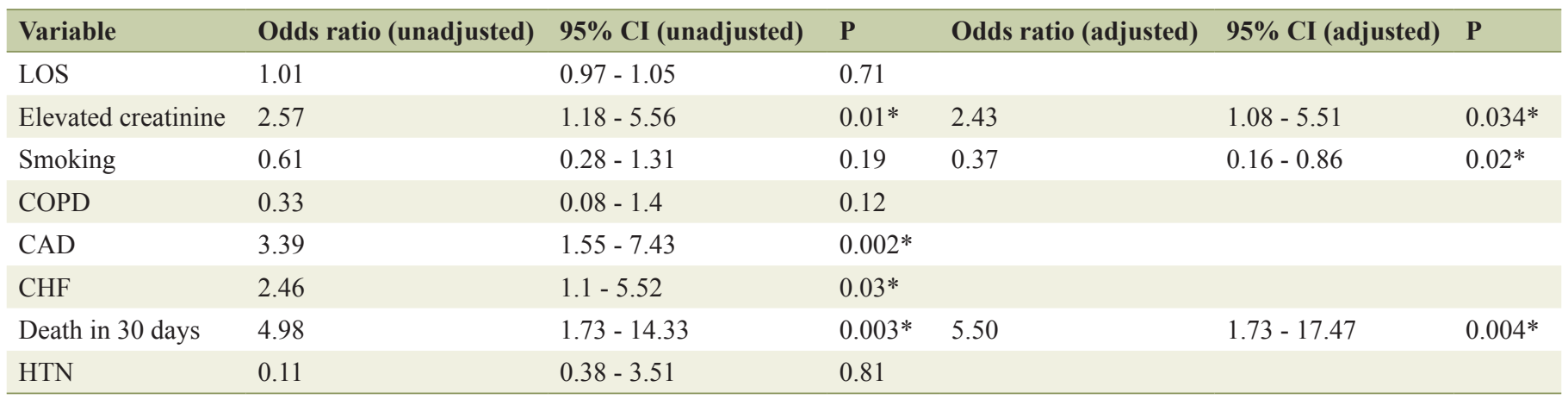


studies with similar end points have reported uniform results. $\mathrm{Wu}$ et al reported an LOS of 8.73 versus 6.34 days in patients with elevated troponin which is comparable difference reported by our study [11]. The study by Vasile et al detected an increased long-term mortality in patients with elevated troponin by measuring troponin in the first $6 \mathrm{~h}$ of admission with AGIB, while our study furthered the findings by following troponins for $24 \mathrm{~h}$ and thus observing a higher short-term mortality in patients with elevated cardiac biomarkers, TnI [10].

Cardiac TnI shares its functionality with troponin $\mathrm{C}$ and $\mathrm{T}$ and is important in regulating the contractions of cardiac musculature. TnI is a more sensitive and specific marker of myocardial injury, sometimes even able to detect cardiac damage with no ECG changes $[13,14]$. Mortality reported in our cohort was a substantial $21 \%$ compared to $5 \%(\mathrm{P}=0.001)$ in patients with and without troponin elevation, respectively. This is concerning specifically to the physicians on the general medical floor managing these patients, as these patients may be at higher risk of cardiovascular damage $[15,16]$. Elevated troponin may signify an MI, and with the concurrent AGIB, endoscopy may be deferred by physicians, even though it has been shown to be safe and indicated in patients even prior to any cardiac intervention [17]. In a study out of the UK, which audited 6,750 patients suffering from AGIB, only 74\% received endoscopy as inpatients, and $50 \%$ of these were within $24 \mathrm{~h}$ of presentation [18]. With the higher short-term mortality identified in our study, measures should be taken to aid physicians in stratifying inpatient or emergent endoscopy by including clinical laboratory variables such as troponin.

Cardiac troponin elevation, in general, has been associated with increased short-term mortality in patients admitted to the ICU [19]. Elevation of troponin can be acute or chronic based on the underlying pathogenesis $[20,21]$. Troponin may be released from the striated muscles of the heart due to either diminished supply or increasing demand from tachycardia. Thus, troponin elevation may act as a surrogate marker for the systemic manifestation of AGIB, such as anemia and hypotension. Anemia was independently shown to cause a higher troponin level with a higher risk of myocardial injury and therefore higher mortality [14, 22].

Despite its high sensitivity and specificity, it may be difficult to interpret a high TnI result in renal failure [23]. The higher levels may be due to the inflammatory state common in renal failure, volume overload or micro-infarctions [24]. Even with renal failure, higher troponin states are accepted as markers for myocardial injury [25]. Acute kidney injury following AGIB was associated with longer hospital stay and increased cost burden [26]. Our study controlled for these variables including renal failure, coronary artery disease and heart failure. Even on multivariate analysis, troponin elevation was still found to be a prognostic marker for increased mortality in patients with AGIB.

With these findings, the authors conclude that serum troponin levels are an important determinant of prognosis for patients with an AGIB. Elevated troponins may be directly related to high-risk stigmata, mortality, LOS, and need for endoscopic evaluation. The authors highly recommend trending troponins during admissions for any patients with an AGIB and thus help in stratifying these patients with a need for intervention. Large studies are needed to confirm our current findings.

\section{Limitations of the study}

The design of our study is retrospective. Patient population in the troponin elevation group is small. This may limit the interpretation of our results. A larger study may help to further elucidate troponin elevation with mortality in AGIB patients. Although LOS trended higher in the troponin elevation group, this may be due to high comorbidities in patients in this group.

\section{Conclusion}

Elevated cardiac troponin in AGIB is a marker of poor outcomes and is associated with high short-term mortality. It is also associated with increased LOS and utilization of resources. Hence it can be used as a marker in risk stratifying patients with AGIB to prevent morbidity and mortality associated with this condition.

\section{Significance of this study}

\section{What is already known about this subject?}

Troponin elevation is generally considered as a marker of MI. Previous studies showed it can be elevated in critically ill patients in intensive care unit and is associated with increased mortality.

Its role in AGIB has not been well established.

\section{What are the new findings?}

Troponin elevation can be used as a marker to risk stratify patients admitted with AGIB.

Troponin elevation on presentation is associated with increased short-term mortality and longer duration of hospital stay.

How might these results change the focus of research or clinical practice?

GBS and Rockall score are currently used in clinical practice to predict mortality of patients presenting with AGIB. Studies can be done on addition of troponin levels to these clinical scores to predict the outcomes of these patients.

\section{Acknowledgments}

Bassett Research Institute, Bassett Medical Center, Cooperstown, NY 13326. 


\section{Conflicts of Interest}

The authors declare that they have no conflicts of interest.

\section{References}

1. Peery AF, Dellon ES, Lund J, Crockett SD, McGowan CE, Bulsiewicz WJ, Gangarosa LM, et al. Burden of gastrointestinal disease in the United States: 2012 update. Gastroenterology. 2012;143(5): 1179-87.e1-3.

2. Abougergi MS, Travis AC, Saltzman JR. The in-hospital mortality rate for upper GI hemorrhage has decreased over 2 decades in the United States: a nationwide analysis. Gastrointest Endosc. 2015;81(4):882-888 e881.

3. Katschinski BD, Logan RF, Davies J, Langman MJ. Audit of mortality in upper gastrointestinal bleeding. Postgrad Med J. 1989;65(770):913-917.

4. Ruigomez A, Garcia Rodriguez LA, Hasselgren G, Johansson S, Wallander MA. Overall mortality among patients surviving an episode of peptic ulcer bleeding. J Epidemiol Community Health. 2000;54(2):130-133.

5. Alpert JS, Thygesen K, Antman E, Bassand JP. Myocardial infarction redefined--a consensus document of The Joint European Society of Cardiology/American College of Cardiology Committee for the redefinition of myocardial infarction. J Am Coll Cardiol. 2000;36(3):959-969.

6. Kaya E, Karaca MA, Aldemir D, Ozmen MM. Predictors of poor outcome in gastrointestinal bleeding in emergency department. World J Gastroenterol. 2016;22(16):42194225 .

7. Gonzalez-Gonzalez JA, Monreal-Robles R, Garcia-Compean D, Paz-Delgadillo J, Wah-Suarez M, MaldonadoGarza HJ. Nonvariceal upper gastrointestinal bleeding in elderly people: Clinical outcomes and prognostic factors. J Dig Dis. 2017;18(4):212-221.

8. Korff S, Katus HA, Giannitsis E. Differential diagnosis of elevated troponins. Heart. 2006;92(7):987-993.

9. Kelley WE, Januzzi JL, Christenson RH. Increases of cardiac troponin in conditions other than acute coronary syndrome and heart failure. Clin Chem. 2009;55(12):20982112.

10. Vasile VC, Babuin L, Rio Perez JA, Alegria JR, Song LM, Chai HS, Afessa B, et al. Long-term prognostic significance of elevated cardiac troponin levels in critically ill patients with acute gastrointestinal bleeding. Crit Care Med. 2009;37(1):140-147.

11. Wu IC, Yu FJ, Chou JJ, Lin TJ, Chen HW, Lee CS, Wu DC. Predictive risk factors for upper gastrointestinal bleeding with simultaneous myocardial injury. Kaohsiung J Med Sci. 2007;23(1):8-16.

12. Cook DJ, Griffith LE, Walter SD, Guyatt GH, Meade MO, Heyland DK, Kirby A, et al. The attributable mortality and length of intensive care unit stay of clinically im- portant gastrointestinal bleeding in critically ill patients. Crit Care. 2001;5(6):368-375.

13. Nunes JP. Cardiac troponin I in systemic diseases. A possible role for myocardial strain. Rev Port Cardiol. 2001;20(7-8):785-788.

14. Bellotto F, Fagiuoli S, Pavei A, Gregory SA, Cati A, Silverj $\mathrm{E}$, Plebani $\mathrm{M}$, et al. Anemia and ischemia: myocardial injury in patients with gastrointestinal bleeding. Am J Med. 2005;118(5):548-551.

15. Cappell MS. A study of the syndrome of simultaneous acute upper gastrointestinal bleeding and myocardial infarction in 36 patients. Am J Gastroenterol. 1995;90(9):1444-1449.

16. Emenike E, Srivastava S, Amoateng-Adjepong Y, alKharrat T, Zarich S, Manthous CA. Myocardial infarction complicating gastrointestinal hemorrhage. Mayo Clin Proc. 1999;74(3):235-241.

17. Yachimski P, Hur C. Upper endoscopy in patients with acute myocardial infarction and upper gastrointestinal bleeding: results of a decision analysis. Dig Dis Sci. 2009;54(4):701-711.

18. Hearnshaw SA, Logan RF, Lowe D, Travis SP, Murphy MF, Palmer KR. Use of endoscopy for management of acute upper gastrointestinal bleeding in the UK: results of a nationwide audit. Gut. 2010;59(8):1022-1029.

19. Vasile VC, Chai HS, Abdeldayem D, Afessa B, Jaffe AS. Elevated cardiac troponin $\mathrm{T}$ levels in critically ill patients with sepsis. Am J Med. 2013;126(12):1114-1121.

20. Zethelius B, Johnston N, Venge P. Troponin I as a predictor of coronary heart disease and mortality in 70-yearold men: a community-based cohort study. Circulation. 2006;113(8):1071-1078.

21. Khan NA, Hemmelgarn BR, Tonelli M, Thompson CR, Levin A. Prognostic value of troponin T and I among asymptomatic patients with end-stage renal disease: a meta-analysis. Circulation. 2005;112(20):3088-3096.

22. Ralli S, Horwich TB, Fonarow GC. Relationship between anemia, cardiac troponin I, and B-type natriuretic peptide levels and mortality in patients with advanced heart failure. Am Heart J. 2005;150(6):1220-1227.

23. Choy JB, Armstrong PW, Ulan RA, Campbell PM, Gourishankar S, Prosser CI, Tymchak WJ. Do cardiac troponins provide prognostic insight in hemodialysis patients? Can J Cardiol. 2003;19(8):907-911.

24. Wang AY, Lai KN. Use of cardiac biomarkers in endstage renal disease. J Am Soc Nephrol. 2008;19(9):16431652.

25. Freda BJ, Tang WH, Van Lente F, Peacock WF, Francis GS. Cardiac troponins in renal insufficiency: review and clinical implications. J Am Coll Cardiol. 2002;40(12):2065-2071.

26. Cakmak U, Merhametsiz O, Gok Oguz E, Ercan Z, Haspulat A, Ozkan SK, Canbakan B, et al. Effects of acute kidney injury on clinical outcomes in patients with upper gastrointestinal bleeding. Ren Fail. 2016;38(2):176-184. 